\title{
EXAMINATION OF THICK-FILMS USING MODERN SURFACE ANALYTICAL TECHNIQUES ${ }^{\dagger}$
}

\author{
G. HARSÁNYI ${ }^{\dagger}$ and G. RIPKA \\ Industrial Research Institute for Electronics, Budapest, Hungary. Technical University, Budapest, Hungary
}

(Received November 1, 1982; in final form June 6, 1983)

Modern surface analytical methods/EMPA, AES, SIMS etc. were used for studying the different layers in thickfilm integrated circuits. Diffusion and migration effects, surface impurity distributions and surface compositions were examined. Some of the results are presented in this paper. Electrical measurements are not discussed here; only examples of the practical use of the methods are demonstrated.

\section{INTRODUCTION}

The hybrid microelectronics industry today is dominated by thick film. Some of the many advantages of thick film technology are grouped into four major categories of easy production, flexibility, reliability and economy.

Thick film technology is used in many socialist countries, but there is not enough information about the pastes and the reaction between the layers. In general, the exact composition of pastes is not known. As is wellknown, the composition and microstructure of thick film layers are very complicated.

The examination of thick film using modern surface analytical techniques is now being studied (in cooperation with a number of Hungarian research institutes: HIKI; TKI; GKL; FKI; Tech.Univ.Bp.Phys.Inst.). In the following paper a survey of this work is given. Our aim is to render a brief report on the methods applied in the course of our investigations and to describe the nature of the results obtained.

\section{DIFFUSION AND MIGRATION EFFECTS} (SEM, EMPA - Electron Microprobe Analysis)

The electrochemical migration of silver which takes place in conductor-insulatorconductor systems is a phenomenon known for a long time. ${ }^{1}$ The essence of this phenomenon is that in a wet medium, upon the application of voltage, a migration of ions starts between the two electrodes and owing to the formation of dendrite shortcircuits are created. Investigations in past years have shown also that in the case of silverfree thick-layer pastes migration effects must be reckoned with. ${ }^{2}$ This means at the same time that compositions inclined to migration must be avoided in circuits of precision design and high reliability.

In the course of our research work we carried out parameter measurements and microanalytical examinations following climatic loads. We followed the changes by microscope first, then a scanning electron microscope, as a means of making a wavelength-dispersive analysis of the emitted X-rays. By making secondary and backscattered electron images, X-ray maps and profiles, it can be determined which components carry out migration at the individual conductors and to what extent. For

\footnotetext{
${ }^{\dagger}$ Paper originally presented at the 5th International Spring Seminar on Electrotechnology held at Prenet, Czechoslovakia, 1-4 June, 1982.
} 


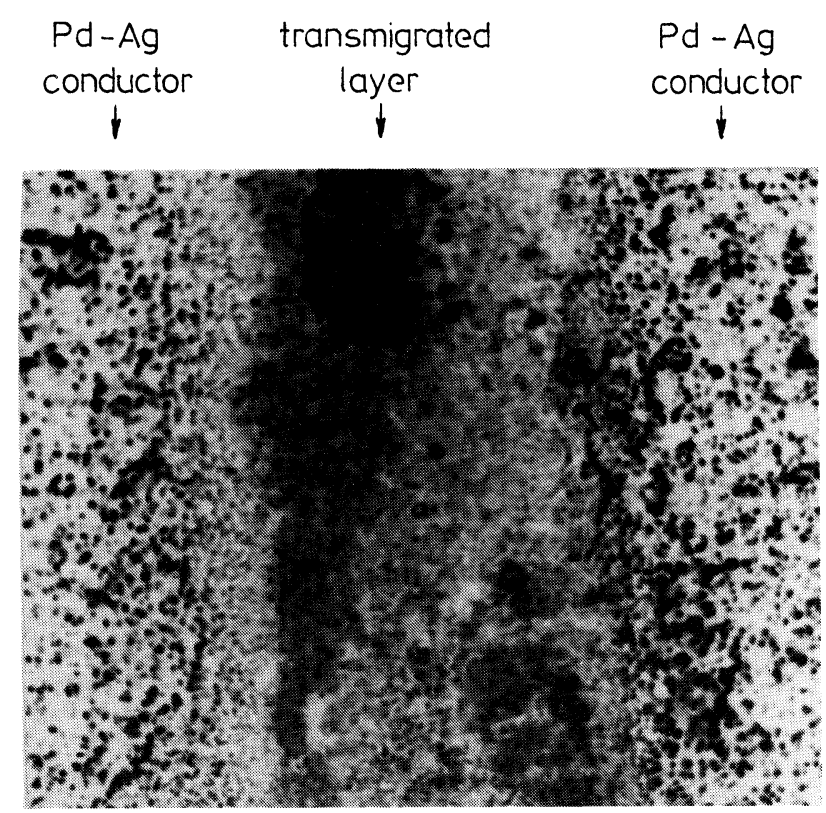

FIGURE $1 \quad$ Back-scattered electron image.

illustration we describe below the results of some of our studies. In Figure 1 - in a backscattered electron image - a transmigrated layer on a ceramic substrate between two $\mathrm{Pd}-\mathrm{Ag}$ conductors can be seen. In Figure 2 the X-ray emission map shows the distribution of silver, while in Figure 3 map palladium emission is given. It appears clean that palladium has not, but on the other hand, silver has, migrated to a great extent between the two conductors.

In Figure 4 the secondary electron image of a high-powered magnification of a copper dendrite has been illustated.

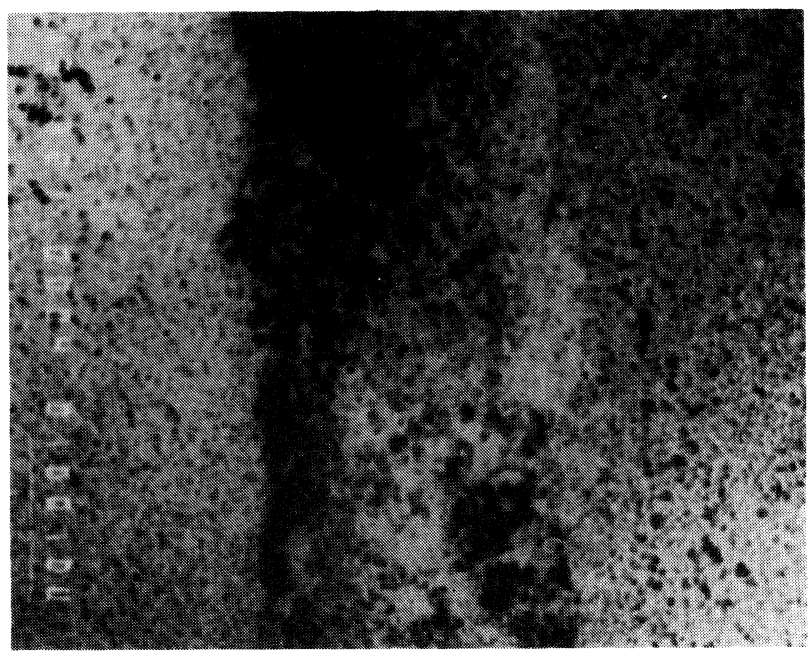

FIGURE $2 \quad \mathrm{AgL}_{\alpha} \mathrm{X}$-ray emission map. 

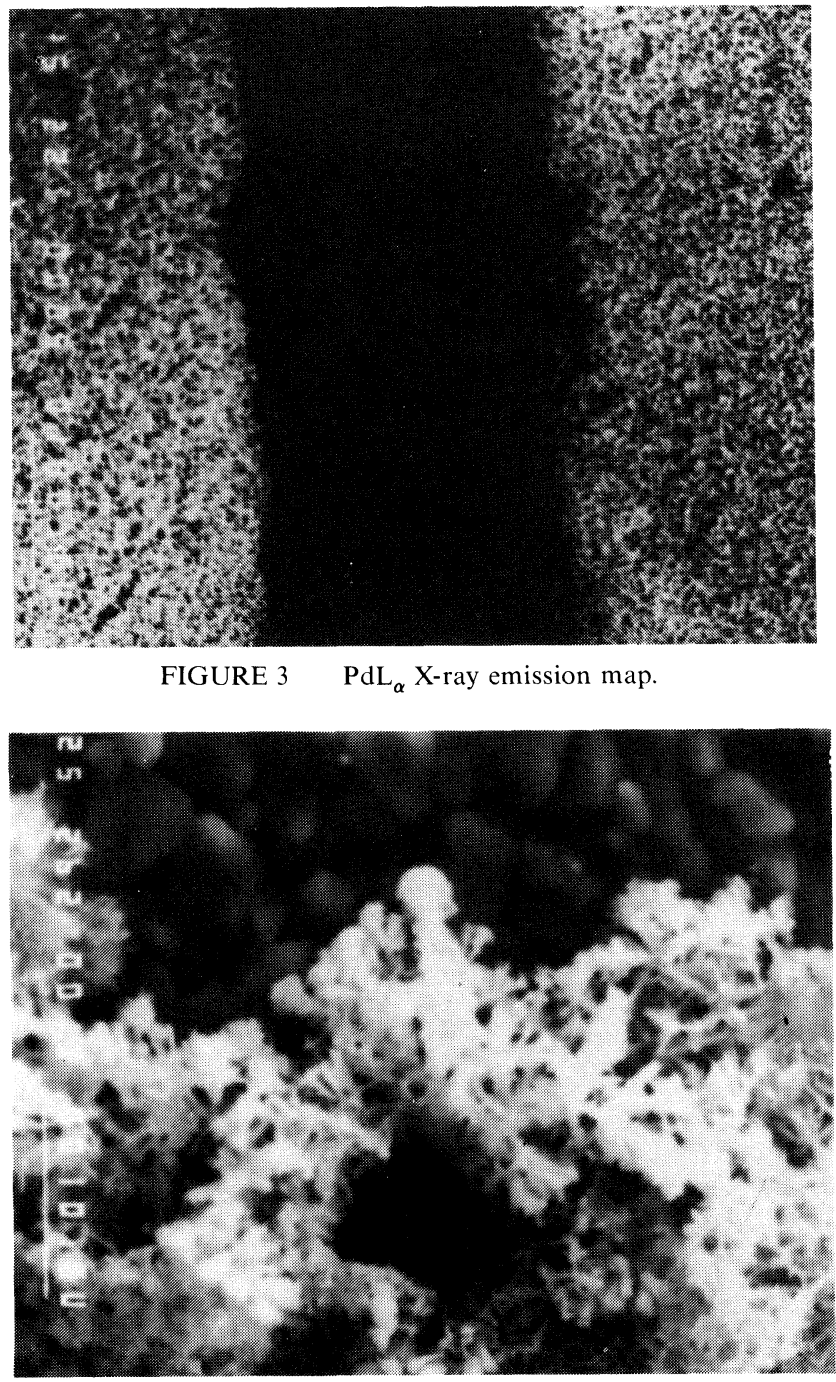

FIGURE 4 SEI of a copper dendrite.

Through our investigations we also discovered that the following elements can migrate: $\mathrm{Ag} ; \mathrm{Au} ; \mathrm{Cu} ; \mathrm{Sn} ; \mathrm{Pb}$.

In addition to electrochemical migration we also examined other material migration phenomena, by the aid of X-ray images. The subject of our studies were plane capacitors formed on substrates of $\mathrm{Al}_{2} \mathrm{O}_{3}$ materials (we used several types of material as conductor, while the dielectric has been $\mathrm{BaTiO}_{3}$ ). Conspicious phenomena were the following:

- Aluminium was traceable in the whole system (even at places far away from the substrate);

- In the upper layer of the substrate an interfacial zone was formed;

- The $\mathrm{Bi}$ component originating from the binding material $\mathrm{Bi}_{2} \mathrm{O}_{3}$ of the dielectric layer of the capacitor can be found in the substrate too; 
- The $\mathrm{Ba}$ penetrates into the conductors and into the interfacial zone of the ceramic.

\section{SURFACE IMPURITY OF CONDUCTORS AND DIELECTRICS}

(AES - Auger Electron Spectroscopy)

Inappropriate burning-in conditions of thick-film materials may result in an incorporation of superficial carbon and sulphur contamination. For the study of this problem we applied Auger spectroscopy combined by ion sputtering. We determined the distribution of the above impurities in depth at various technological adjustments. The results are shown in Figure 5.

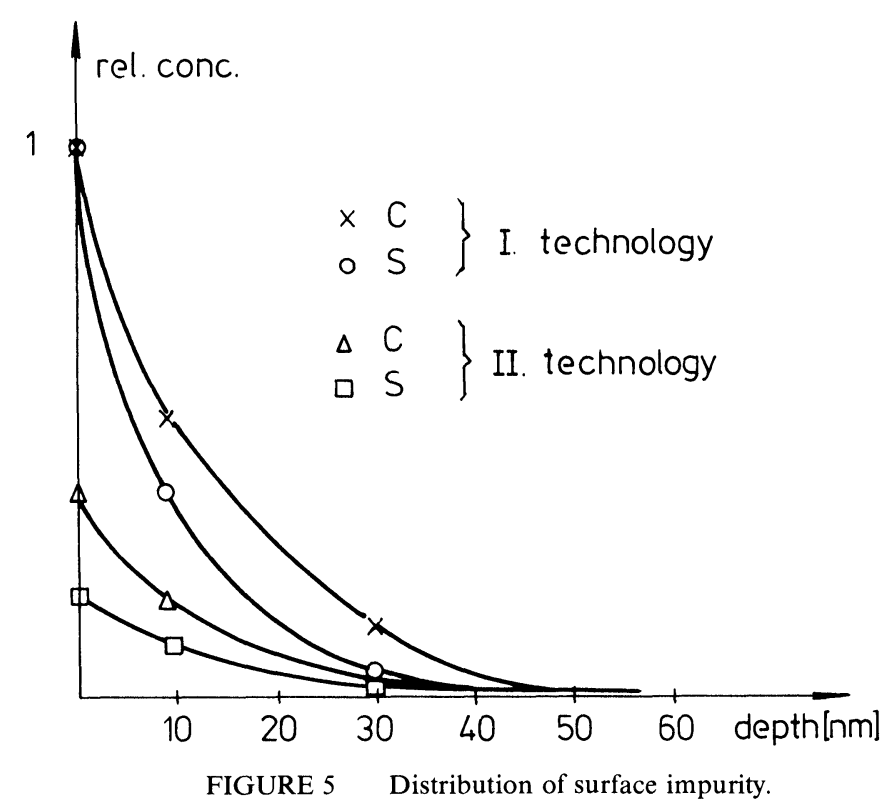

\section{SURFACE COMPOSITION OF CONDUCTORS}

(SIMS - Secondary Ion Mass Spectrometry)

It is important to know the change of composition of conducting layers near to the surface, since diffusion and migration phenomena discussed already in the preceding paragraphs are determined by superficial composition.

A particularly interesting process is the formation of the composition of the Pd-Ag-Pt ternary alloy showing no migration.

We examined the distribution profile in depth of the individual elements with the aid of the SIMS method. The result can be seen in Figure 6.

\section{OXIDATION OF COPPER CONDUCTOR SURFACES}

(AES; LEED - Low Energy Electron Diffraction)

The burn-in of most copper conductors requires a nitrogen atmosphere. The effect of an inappropriate burn-in atmosphere, is however, that an undesirable oxidation of the 


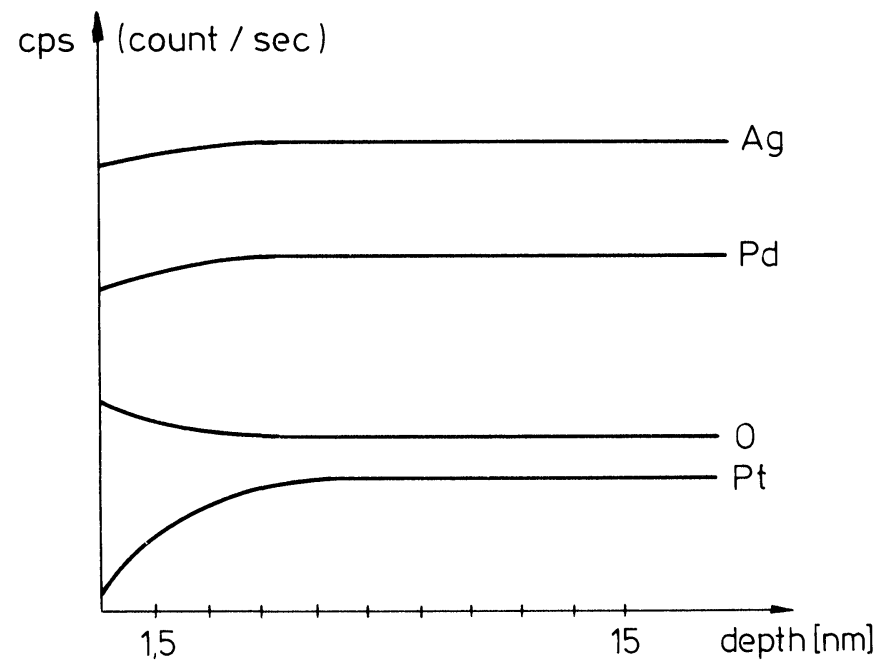

FIGURE 6 SIMS profiles of one conductor layer.

surface can take place. Present methods of centralling the technological process makes it necessary to understand the nature of the connection between the oxide coating and other characteristics (colour, electrical parameters, solder ability, high-frequency features).

\section{REFERENCES}

1. G.T. Kohman, H.W. Hermance, G.H. Downes, Bell System Journal pps 1115-1147, (1955).

2 A. DerMarderosian, pps. 134-136, Proceedings of ISHM 78 

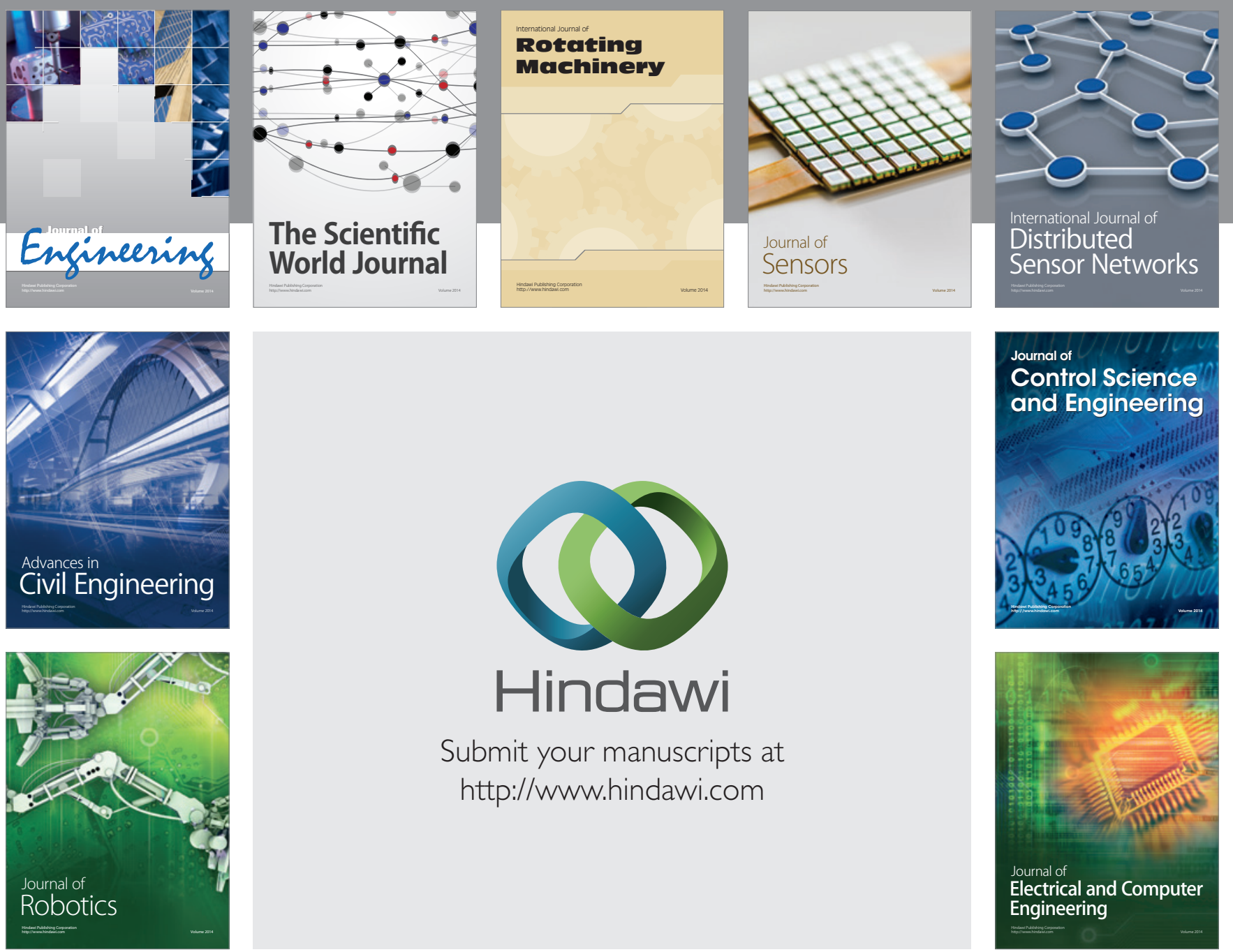

Submit your manuscripts at

http://www.hindawi.com
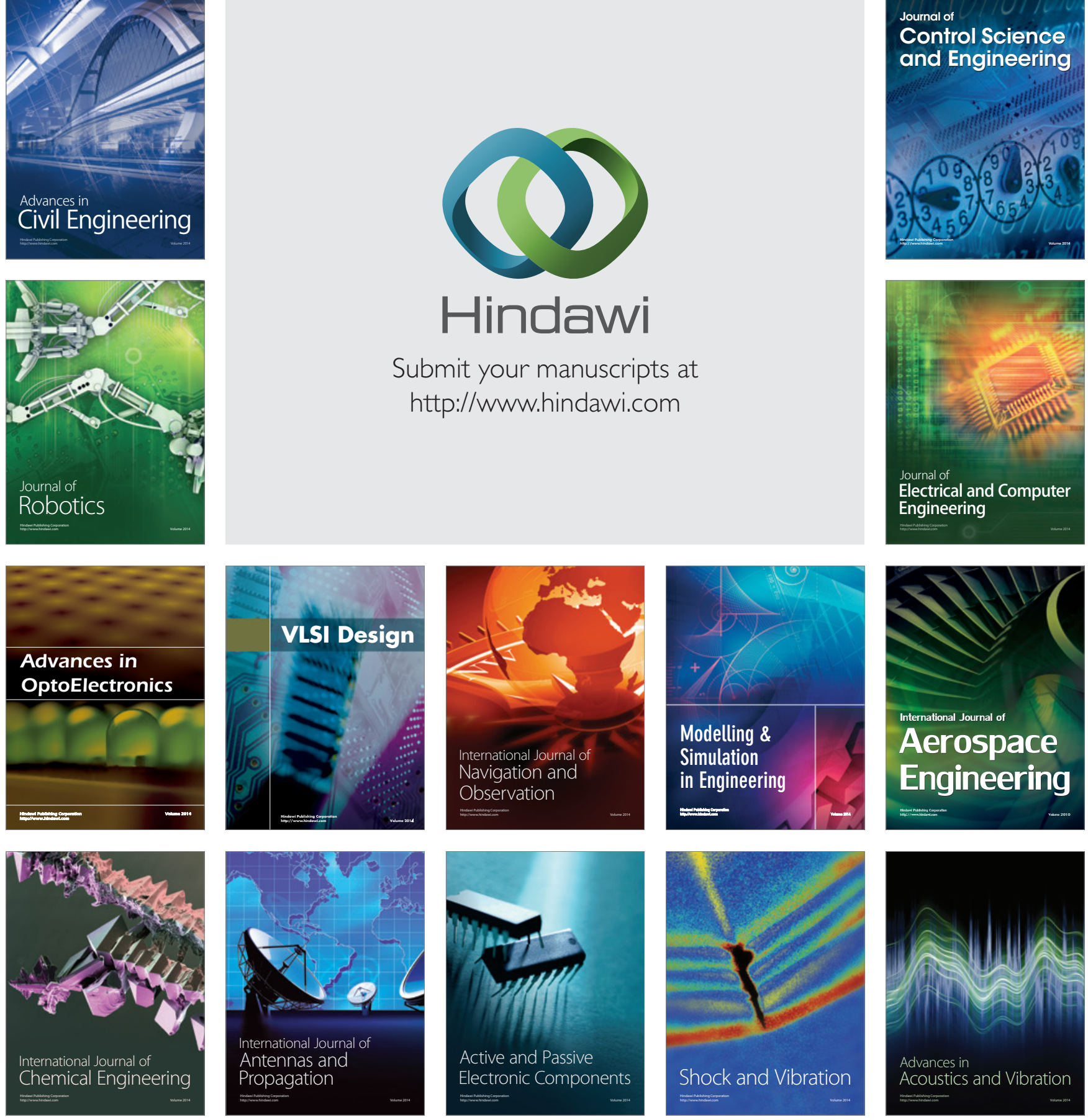